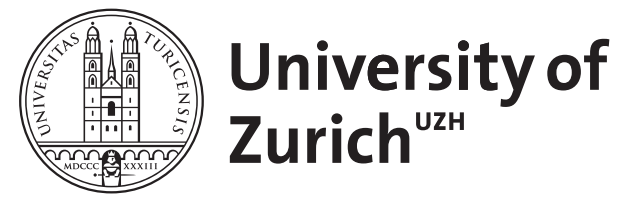

Zurich Open Repository and Archive

University of Zurich

University Library

Strickhofstrasse 39

CH-8057 Zurich

www.zora.uzh.ch

Year: 2013

\title{
Hype um Vitamin D? Nicht während der Schwangerschaft
}

Djalali, Sima

DOI: https://doi.org/10.1024/1661-8157/a001336

Posted at the Zurich Open Repository and Archive, University of Zurich

ZORA URL: https://doi.org/10.5167/uzh-79738

Journal Article

Accepted Version

Originally published at:

Djalali, Sima (2013). Hype um Vitamin D? Nicht während der Schwangerschaft. Praxis, 102(14):879-880.

DOI: https://doi.org/10.1024/1661-8157/a001336 


\section{Hype um Vitamin D? Nicht während der Schwangerschaft}

Frage:

Führt mütterlicher Vitamin-D-Mangel während der Schwangerschaft zu einer langfristig erniedrigten Knochendichte beim Kind?

Hintergrund:

Folsäure, Eisen, Magnesium - die Liste der Supplementierungsempfehlungen für Schwangere ist lang. Uneins war die Literatur bislang, ob auch Vitamin D dazugehören sollte. Eine kleine Studie (<200 Teilnehmer) legte nahe, dass mütterlicher Vitamin-D-Mangel in der Schwangerschaft zu einer verringerten Knochendichte des Kindes führen könne. Weitere Studien zeigten keine Assoziation bzw. sogar eine inverse Beziehung, d.h. Vitamin-D-Mangel der Mutter ziehe eine erhöhte Knochendichte beim Kind nach sich. Die Untersuchung der Zusammenhänge ist epidemiologisch und präventivmedizinisch bedeutsam, da ca. $70 \%$ der ansonsten gesunden Schwangeren heute erniedrigte Vitamin-D-Spiegel aufweisen. Eine prospektive populationsbasierte Kohortenstudie verspricht Aufklärung.

\section{Einschlusskriterien:}

- Schwangere Frauen und ihre Kinder mit Geburtstermin zwischen April 1991 und 31. Dezember 1992

\section{Ausschlusskriterien:}

- Mehrlingsgeburten

- Totgeburten

\section{Studiendesign und Methode:}

Prospektive Kohortenstudie

\section{Studienort:}

England

\section{Interventionen:}

- Messung der mütterlichen 25-Hydroxyvitamin-D-Serumkonzentration während der Schwangerschaft (Verwendung der jeweils letzten Messung, unabhängig vom Schwangerschaftsalter; Statistische Standardisierung der Ergebnisse für die Analyse

- Messung der 25-Hydroxyvitamin-D-Serumkonzentration beim Kind im 9.-10. Lebensjahr

- Knochendichtemessung beim Kind im 9.-10. Lebensjahr

\section{Outcome:}

Pr i mär er Out come

- Ganzkörperknochendichteindex des Kindes (Total Body Less Head, TBLH)

- Knochendichteindex der Wirbelsäule des Kindes

Sekundäre Out comes

- Knochendichteindex des Kindes korrigiert für Standardwerte spezifischer Knochengruppen

- Einfluss von UV-Exposition der Mutter und maternaler 25-Hydroxyvitamin-DSerumkonzentration im dritten Schwangerschaftstrimester auf die Knochendichteindices des Kindes 
- $14^{\prime} 541$ werdende Mütter und ihre Kinder wurden für die Kohorte rekrutiert. Davon nahmen 3960 Mutter-Kind-Paare den Follow-up-Termin zur Knochendichtemessung wahr. Die Kinder waren dabei im Durchschnitt 9,9 Jahre alt.

- Eine Korrelation zwischen maternaler 25-Hydroxyvitamin-D-Serumkonzentration und kindlicher Knochendichte wurde für kein Trimester bestätigt. Die mittleren Knochendichteindices der Kinder waren gleich, unabhängig ob die 25-Hydroxyvitamin-DSerumkonzentration der Mütter während der Schwangerschaft als ausreichend, erniedrigt oder defizitär klassifiziert worden war.

- Ein Einfluss von UV-Exposition der Mutter während des dritten Trimesters auf die Knochendichte wurde nicht belegt.

\title{
Kommentar:
}

- Die statistischen Analysen in dieser Studie berücksichtigen neben Alter der Mutter und Alter und Geschlecht des Kindes eine Vielzahl von Störgrössen, welche die komplexen Einflüsse auf den Vitamin-D-Stoffwechsel von Mutter und Kind sowie die Knochenmineralisation abbilden. Die Wahrscheinlichkeit, dass eine unbekannte Störgrösse eine bestehende, klinisch relevante Korrelation überlagert, ist als gering einzuschätzen.

- Die Anzahl unvollständiger Datensätze in dieser Studie war vergleichsweise gering und fehlende Messwerte wurden durch Imputation vervollständigt, was auch die Wahrscheinlichkeit eines systematischen Selektionsfehlers verringert.

- Diese Studie lässt keine Rückschlüsse darüber zu, ob Vitamin D während der Schwangerschaft andere Vor- oder Nachteile für das Ungeborene hat. Auch ist nicht gesagt, dass der individuelle Vitamin-D-Haushalt des Kindes nicht dessen Knochengesundheit beeinflusst. Hinsichtlich der Knochendichte im späteren Kindesalter besteht nach den vorliegenden Ergebnissen jedoch keine Evidenz für eine Vitamin-D-Supplementierung während der Schwangerschaft.

\section{Literatur:}

Lawlor DA, et al.: Association of maternal vitamin D status during pregnancy with bonemineral content in offspring: a prospective cohort study. Lancet 2013 Mar 18; doi: 10.1016/S0140-6736(12)62203-X. [Epub ahead of print].

\author{
Verfasser: \\ Dr. med. Sima Djalali \\ Institut für Hausarztmedizin \\ Universität Zürich \\ Pestalozzistrasse 24 \\ 8091 Zürich \\ sima.djalali@usz.ch
}

